\title{
Laboratory experiment with Helium-Neon laser: Gain and oscillation spectral widths
}

\section{Práctica de laboratorio con láser de helio-neón: Anchuras espectrales de ganancia y de oscilación}

\author{
Sebastián Jarabo ${ }^{*}$, S) \\ Departamento de Física Aplicada, Facultad de Ciencias, Universidad de Zaragoza, C/ Pedro Cerbuna 12, \\ 50.009 Zaragoza, Spain \\ (*) Email: sjarabo@unizar.es \\ S: miembro de SEDOPTICA / SEDOPTICA member \\ Received / Recibido: 05/03/2014. Revised / Revisado: 11/03/2014. Accepted / Aceptado: 12/03/2014. \\ DOI: http://dx.doi.org/10.7149/OPA.47.1.63
}

\begin{abstract}
:
The relationship between gain and oscillation spectral profiles is studied and analysed from an educational perspective. A theoretical explanation based on simple models is presented. Expressions of spectral profiles and widths are deduced by means of this theory. Spectral profiles and widths are measured with a laboratory experiment based on low-cost lasers. Theoretical and experimental values are in agreement.
\end{abstract}

Key words: Optics Experiments, Optics Education, Laser, Optical Amplification, Gain Spectral Profile, Oscillation Spectral Profile.

\section{RESUMEN:}

La relación entre los perfiles de ganancia y de oscilación de un láser es estudiada y analizada desde una perspectiva docente. Se presenta una explicación teórica basada en modelos sencillos. A partir de esta teoría, se formulan dichos perfiles espectrales y se relacionan sus anchuras. Los perfiles y las anchuras son medidos en una práctica de laboratorio realizada con láseres de bajo costo. La comparación entre teoría y experimento resulta satisfactoria.

Palabras clave: Prácticas de Óptica, Educación en Óptica, Láser, Amplificación Óptica, Perfil Espectral de Ganancia, Perfil Espectral de Oscilación.

\section{REFERENCES AND LINKS / REFERENCIAS Y ENLACES}

[1]. C. Lavieja, S. Jarabo, "Láser de helio-neón: Prácticas de laboratorio sobre láser e interacción luzmateria", Opt. Pura Apl. 44, 347-359 (2011).

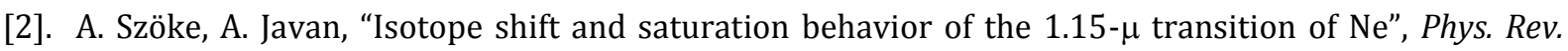
Lett. 10, 521-524 (1963). DOI

[3]. R. L. Fork, D. R. Herriott, H. Kogelnik, "A scanning spherical mirror interferometer for spectral analysis of laser radiation", Appl. Opt. 3, 1471-1484 (1964). DOI

[4]. M. Hercher, "Spherical mirror Fabry-Pérot interferometer", Appl. Opt. 7, 951-966 (1968). DOI

[5]. E. K. Hasle, "Polarization properties of He-Ne-lasers", Opt. Commun. 31, 206-210 (1979). DOI

[6]. L. W. Davis, G. Patsakos, "A spherical mirror Fabry-Pérot-interferometer for microwave demonstrations", Am. J. Phys. 55, 917-920 (1987). DOI

[7]. P. Nachman, A. C. Bernstein, "Scanning, spherical-mirror Fabry-Pérot interferometer: An upperdivision optics laboratory experiment", Am. J. Phys. 65, 202-213 (1997). DOI

[8]. M. Jackson, D. Bauen, J. E. Hasbun, "Investigation of laser fundamentals using a helium-neon laser", Eur. J. Phys. 22, 211-218 (2001). DOI

[9]. N. Whitlatch, "Spectrum analysis of optical resonators", Am. J. Phys. 58, 556-560 (1990). DOI 
[10]. Spectra-Physics Laser Technical Bulletin Number 6, "Scanning Spherical-Mirror Interferometers for the Analysis of Laser Mode Structure" (1976).

[10]. O. Svelto, Principles of Lasers, Plenum Press, New York (1998).

[11]. W. T. Silfvast, Laser Fundamentals, Cambridge University Press (2004).

[12]. H. J. Döhner, W. Elsässer, "Analysis of a four-level laser system: investigations of the output power characteristics of a He-Ne laser", Am. J. Phys. 59, 327-330 (1991). DOI

[13]. K. L. Vander Sluis, G. K. Werner, P. M. Griffin, H. W. Morgan, O. B. Rudolph, P. A. Staats, "A simplified construction of a Helium-Neon visible laser", Am. J. Phys. 33, 225-240 (1965). DOI

\section{Introducción}

En el artículo "Láser de helio-neón: Prácticas de laboratorio sobre láser e interacción luzmateria" [1] se presenta de forma detallada un amplio conjunto de prácticas de laboratorio realizadas con tubos y láseres de helio-neón, las cuales pueden ser adaptadas tanto a estudios de grado como de máster. Como ya se indicó en dicha referencia, se pueden llevar a cabo muchas más prácticas docentes empleando tubos y láseres de helio-neón. Entre éstas, prácticas que ilustren las diferencias entre los perfiles espectrales de ganancia y de oscilación, aunque ambos estén íntimamente relacionados y, en especial, que el perfil de oscilación suele ser más estrecho que el perfil de ganancia. Lamentablemente, resulta muy difícil encontrar referencias bibliográficas que muestren claramente dichos perfiles [2], aunque sí se encuentra un número mayor de artículos que contienen medidas de los modos longitudinales de emisión de láseres de helio-neón [3-9].

En la Ref. [1] se determinó experimentalmente la anchura del perfil de oscilación de un láser de helio-neón (emitiendo en $632.8 \mathrm{~nm}$ ). Aunque el resultado obtenido es razonable, hay que reconocer que el procedimiento seguido no puede considerarse demasiado fiable. Además, en dicha referencia también se midió la ganancia media de un tubo de helio-neón, pero no se intentó medir el perfil espectral de ganancia. Así pues, en este artículo se presentan medidas orientadas a extraer información sobre los perfiles espectrales de ganancia y de oscilación de un láser de helio-neón. El material de laboratorio utilizado es, básicamente, el mismo que se empleó en la Ref. [1]. El método de medida, bien conocido $[3,4,6-8,10]$ desde los años 60, se basa en el análisis espectral de la emisión del láser de helio-neón mediante un interferómetro Fabry-Pérot de espejos esféricos.

\section{Explicación teórica}

El láser de helio-neón oscilando en la transición de $632.8 \mathrm{~nm}$ se comporta según un esquema de 4 niveles ideal [11-13]. El mecanismo de ensanchamiento que predomina en dicha transición es inhomogéneo (Doppler). Por tanto, el perfil espectral de la sección eficaz de emisión de esta transición, $\sigma(v)$, puede expresarse en buena aproximación mediante una función gaussiana, en la forma:

$$
\begin{gathered}
\sigma(v)=\sigma\left(v_{0}\right) \exp \left(-\vartheta^{2}\right), \\
\vartheta=\frac{v-v_{0}}{\Delta v},
\end{gathered}
$$

donde $v$ representa a la frecuencia óptica, $v_{0}$ es la frecuencia óptica central de la transición y $\Delta v$ es la semianchura del perfil espectral a $1 / e$ de su valor máximo. Entonces, la ganancia (por paso) del tubo de helio-neón podemos expresarla de acuerdo con la expresión [12-13]:

$$
G(v)=\exp (\sigma(v) N L),
$$

siendo $L$ la longitud del medio activo y $N$ la inversión de población de la transición óptica. Por tanto, cuanto mayor es la probabilidad de bombeo, mayor es la inversión de población y mayor es la ganancia. Como la condición necesaria para conseguir emisión láser viene dada por la ecuación:

$$
\ln (G(v))=\alpha,
$$

donde $\alpha$ son las pérdidas (logarítmicas por paso) totales de la cavidad, se suele hablar de ganancia refiriéndose en realidad al logaritmo de la ganancia. Así, la condición anterior se expresa como que la ganancia (logarítmica por paso) debe compensar las pérdidas (logarítmicas por paso) totales de la cavidad. Teniendo en cuenta las Ecs. (1-2) podemos transformar la Ec. (3) en:

$$
\ln (G(v))=\ln \left(G\left(v_{0}\right)\right) \exp \left(-\vartheta^{2}\right) .
$$


Por tanto, la ganancia (logarítmica) responde a un perfil espectral gaussiano de semianchura $\Delta v$ a $1 / e$ de su valor máximo (el mismo que el de la sección eficaz).

Asumiremos que la emisión del láser es siempre monomodo transversal, concretamente $\mathrm{TEM}_{00}$, tal como suele ser la situación habitual. Como en la transición predomina el ensanchamiento inhomogéneo, el láser podría llegar a emitir en todas aquellas frecuencias ópticas del perfil de ganancia para las que la ganancia compensase las pérdidas y sólo en dichas frecuencias. La potencia emitida tendría entonces un perfil espectral distinto al de ganancia, aunque ambos perfiles estarán relacionados entre sí. Al perfil espectral de la potencia emitida se le denomina perfil espectral de oscilación. Pero además, las frecuencias para las que puede llegar a emitirse potencia láser deben coincidir con las frecuencias de los modos longitudinales del resonador. Por tanto, el espectro de la potencia emitida tiene un perfil tipo peine con una envolvente dada por el perfil de oscilación. Tanto el número de modos longitudinales que están oscilando simultáneamente como el perfil de oscilación dependerán del bombeo, ya que el bombeo modifica la inversión de población de la transición y, por tanto, su ganancia.

En la Fig. 1 puede apreciarse cómo el número de modos longitudinales que consiguen oscilar simultáneamente aumenta conforme aumenta la ganancia del medio activo. La función de transmisión espectral del resonador se representa en color naranja, las pérdidas totales de la cavidad en color marrón y el perfil espectral de ganancia (en realidad, de la sección eficaz) cuando compensa las pérdidas en una única frecuencia (en el caso representado, la frecuencia central del espectro) en color azul. En esta situación, la emisión del láser se produciría en un único modo longitudinal. Si aumenta el bombeo, la ganancia crece $\mathrm{y}$, como el ensanchamiento es inhomogéneo, puede superar las pérdidas en aquellas frecuencias para las que no se produce oscilación láser. Y si el bombeo es lo suficientemente alto, empezarán a oscilar varios modos longitudinales (Fig. 1(a)). No obstante, la ganancia para aquellas frecuencias
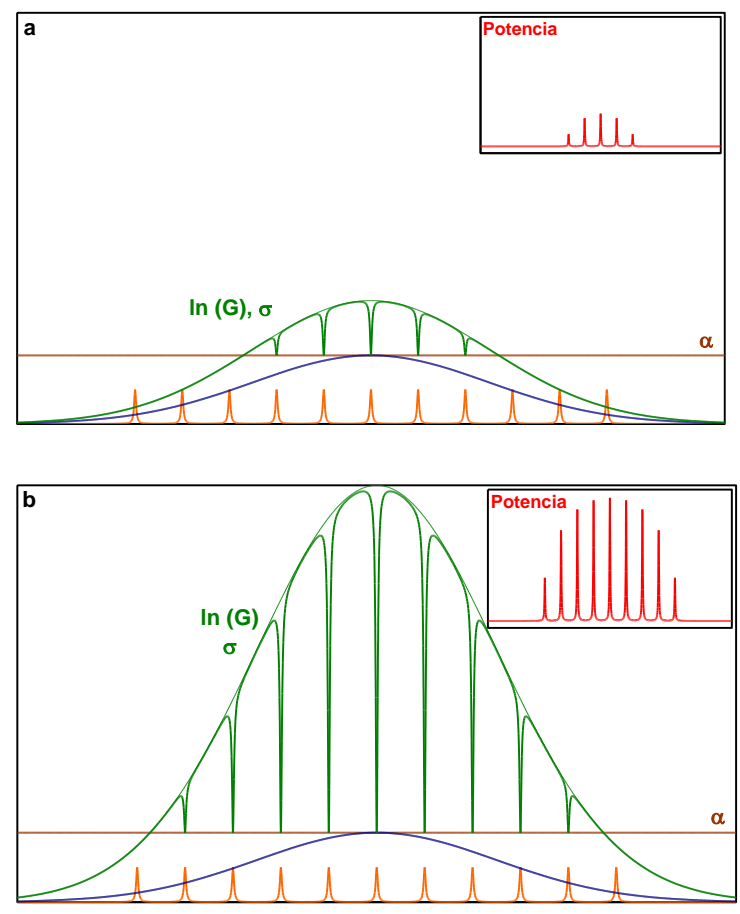

\section{Frecuencia óptica}

Fig. 1. Emisión láser en varios modos longitudinales. (a) Como el ensanchamiento es inhomogéneo, la ganancia puede superar el valor necesario para compensar las pérdidas y se produce oscilación láser en algunos modos longitudinales. (b) Conforme aumenta el bombeo, aumenta el número de modos longitudinales que consiguen oscilar de forma que la anchura espectral del perfil de oscilación puede llegar a superar a la anchura espectral del perfil de ganancia.

donde se produce oscilación queda anclada al valor que compensa las pérdidas de la cavidad. Entonces, el perfil de ganancia responde a una función gaussiana con unos agujeros mucho más estrechos centrados justo en dichas frecuencias (hole-burning espectral [12-13]). Aumentando aún más el bombeo (Fig. 1(b)) el espectro de ganancia sigue aumentando de la misma manera y el número de modos longitudinales sigue aumentando. Por tanto, si el bombeo puede ser lo suficientemente alto, la anchura del perfil de oscilación puede llegar a superar a la anchura espectral de la sección eficaz.

Es posible conocer el perfil de oscilación si determinamos la potencia emitida en cada frecuencia óptica, $P_{s}(v)$, en función de la probabilidad de bombeo $R_{p}$. Como el ensanchamiento es inhomogéneo, podemos tratar dicha potencia de forma independiente para cada frecuencia $y$, por tanto, 


$$
P_{S}(v) \propto R_{p}-R_{p}^{t h}(v) .
$$

Es decir, la potencia de salida aumenta de forma lineal con la probabilidad de bombeo a partir de una determinada probabilidad de bombeo umbral, $R_{p}^{\text {th }}(v)$, la cual es la mínima probabilidad de bombeo necesaria para que la ganancia compense las pérdidas de la cavidad. Así pues, debe ser inversamente proporcional a la sección eficaz lo que nos permite expresar su dependencia funcional con la frecuencia óptica en la forma:

$$
R_{p}^{t h}(v)=R_{p}^{t h}\left(v_{0}\right) \exp \left(\vartheta^{2}\right) .
$$

Y, teniendo en cuenta las Ecs. (6) y (7), el perfil espectral de oscilación normalizado a altura unidad vendrá dado por la expresión:

$$
\frac{P_{S}(v)}{P_{S}\left(v_{0}\right)}=\frac{1}{X_{P}-1}\left[X_{p}-\exp \left(\vartheta^{2}\right)\right],
$$

donde el parámetro

$$
X_{p}=\frac{R_{p}}{R_{p}^{t h}\left(v_{0}\right)},
$$

debe ser mayor que la unidad. Es necesario indicar que ni $R_{p}$ ni $R_{p}^{\text {th }}\left(v_{0}\right)$ pueden ser medidos de forma directa, ya que, entre otros factores, dependen de la geometría de la descarga. La magnitud que sí puede medirse es la intensidad eléctrica $I_{p}$ de la descarga en el tubo. Sin embargo, la Ec. (6) no puede expresarse en función de $I_{p}$ ya que, a intensidades altas, $R_{p}$ e $I_{p}$ dejan de ser proporcionales entre sí $[13,14]$.

En la Fig. 2 se compara el perfil espectral de ganancia (sección eficaz) con el de oscilación para algunos valores del parámetro $X_{P}$. El máximo valor empleado para $X_{P}$ se ha limitado a 10 , tras considerar los valores encontrados en la bibliografía $[2,13,14]$.

La semianchura del perfil de oscilación a $1 / e$ de su valor máximo resulta ser:

$$
\Delta v_{o s c}=\Delta v \sqrt{\ln \left[1+X_{p}(e-1)\right]-1},
$$

de forma que crece con $X_{p}$ e incluso llega a superar a $\Delta v$ siempre que $X_{p}>1+e$ (Fig. 3). Como esta condición no suele verificarse en los láseres de helio-neón $[13,14]$, habitualmente se considera que el espectro de oscilación no llega a ensancharse más que el de ganancia.

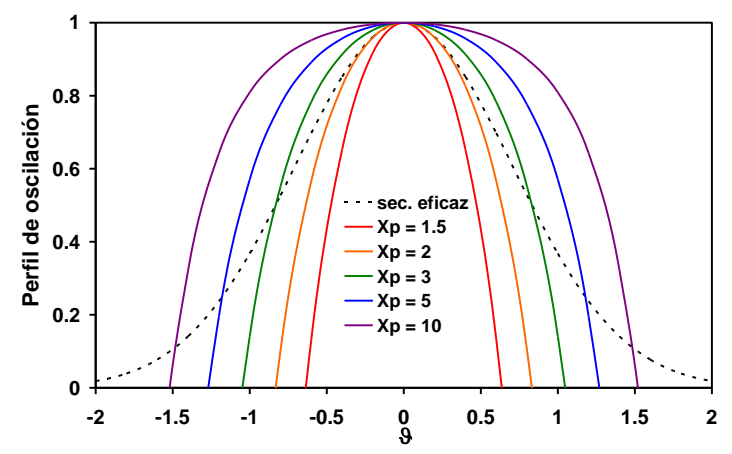

Fig. 2. Perfil espectral de oscilación normalizado a altura unidad para algunos valores del parámetro $X_{p}$.

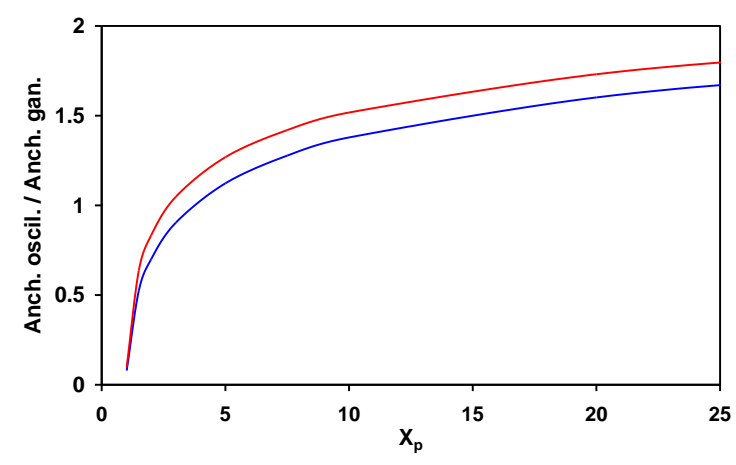

Fig. 3. Cociente entre las anchuras del perfil de oscilación (línea azul: a 1/e del máximo; línea roja: anchura máxima) y del perfil de ganancia en función del parámetro $X_{p}$.

Además, la Ec. (8) sólo tiene sentido físico (valores de potencia no negativos) cuando:

$$
X_{p}>\exp \left(\vartheta^{2}\right)
$$

por lo que la semianchura máxima resulta ser:

$$
\Delta v_{o s c, \max }=\Delta v \sqrt{\ln X_{p}},
$$

la cual superará a $\Delta v$ cuando $X_{p}>e$ (Fig. 3).

La validez de la Ec. (8) puede ser comprobada a partir de la medida del espectro de la potencia emitida por un láser de helio-neón ajustando los valores de $X_{p}$ y de $\Delta v$. Sin embargo, resultará complicado encontrar unos valores únicos de ajuste para ambos parámetros porque sólo se dispone de un único espectro, ya que no es fácil modificar de forma controlada el valor de $X_{p}$. No obstante, esta situación puede ser solucionada si se dispone de un tubo adicional de helio-neón que sea similar al tubo del láser empleado. Para ello, la intensidad eléctrica de la descarga y las presiones de helio y de neón deben ser muy parecidas para que la anchura 


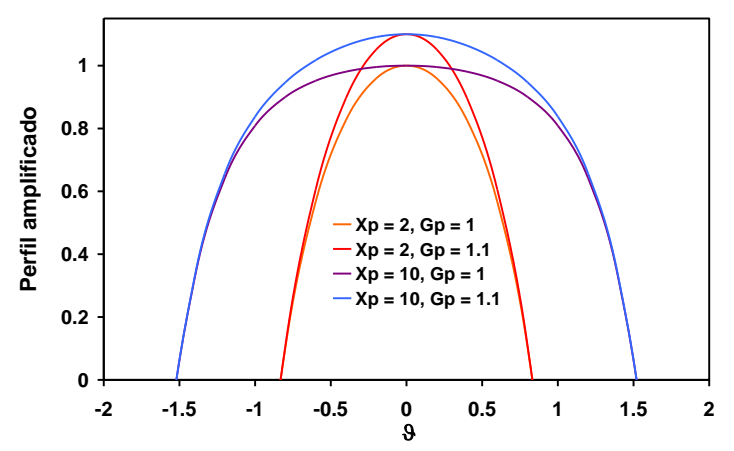

Fig. 4. Perfil de oscilación $\left(G_{p}=1\right)$ y perfil de oscilación amplificado $\left(G_{p}=1.1\right)$ para dos valores representativos de $X_{P} . G_{p}$ denota al valor máximo del espectro de ganancia.

espectral de la sección eficaz apenas varíe. Si la potencia emitida por el láser atraviesa el tubo (bombeado), ésta será amplificada y, por tanto, la potencia a la salida del tubo, $P_{g}(v)$, tendrá un perfil espectral de la forma:

$$
\frac{P_{g}(v)}{P_{s}\left(v_{0}\right)}=\frac{G(v)}{X_{p}-1}\left[X_{p}-\exp \left(\vartheta^{2}\right)\right],
$$

con la ganancia $G(v)$ definida por la Ec. (5). En la Fig. 4 se representan el perfil de oscilación y el perfil de oscilación amplificado para dos valores representativos de $X_{p}$, considerando que el valor máximo del espectro de ganancia es 1.1 (valor compatible con la Ref. [1]) y que $\Delta v=1 \mathrm{GHz}$ (valor compatible con las Refs. [9] y [10]). Ajustando ambos espectros a las Ecs. (8) y (13) debe ser posible determinar correctamente los parámetros $X_{P}$ y $\Delta v$.

\section{Resultados experimentales}

En la Fig. 5 se representa el montaje experimental empleado. La potencia emitida por un láser de helio-neón (Spectra Physics Stability, modelo 120, $632.8 \mathrm{~nm}$ ) es amplificada por un tubo de helio-neón (Spectra Physics, igual al del láser empleado, pero sin espejos) y analizada espectralmente mediante un interferómetro de Fabry-Pérot confocal (Spectra Physics, modelo 470-03, intervalo espectral libre $2 \mathrm{GHz}$, resolución $13 \mathrm{MHz}$ ). El detector utilizado es un fotodiodo de silicio (Thorlabs DET10A/M). Para conseguir que la influencia de la potencia de emisión espontánea procedente del tubo fuese despreciable se colocó un filtro espectral

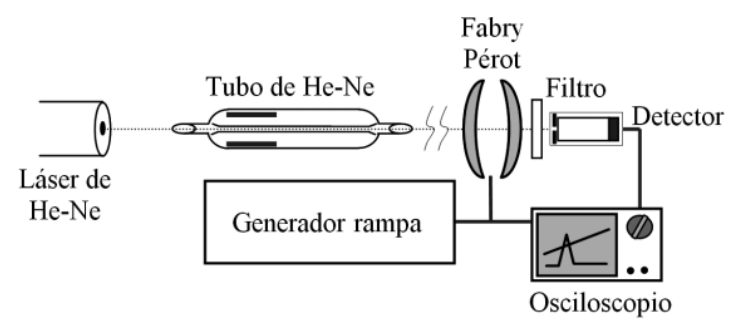

Fig. 5. Montaje experimental.

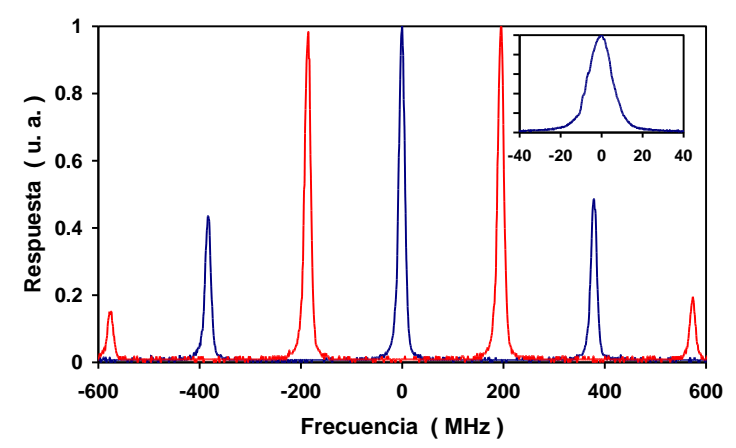

Fig. 6. Análisis espectral de la potencia emitida por el láser de helio-neón. Pueden oscilar simultáneamente 3 (línea azul) ó 4 modos longitudinales (línea roja).

centrado en $633 \mathrm{~nm}$ (Thorlabs, FWHM=3 nm) delante del detector $\mathrm{y}$, además, se procuró que la separación entre el tubo y el detector fuese amplia (75 cm, aproximadamente).

En la Fig. 6 se presenta un ejemplo del espectro de emisión del láser empleado. Emite simultáneamente 3 ó 4 modos longitudinales dentro del perfil de oscilación. El número de modos longitudinales cambia porque su posición no se mantiene fija, sino que se desplaza continuamente a lo largo del perfil de oscilación. No obstante, la separación espectral entre dos modos longitudinales consecutivos se mantiene constante (381 MHz). Midiendo la anchura de los picos a media altura de su valor máximo (ver detalle dentro de la Fig. 6) se determina que la resolución del sistema de medida es $13.5 \mathrm{MHz}$.

Registrando el espectro de emisión sólo podemos medir el perfil de oscilación en unas pocas frecuencias. Sin embargo, sí es posible medir el perfil de oscilación aprovechando que los modos longitudinales van corriendo a lo largo del perfil y registrando espectros de emisión durante un tiempo suficientemente largo (manteniendo la persistencia de la pantalla de un osciloscopio digital, por ejemplo). 
En la Fig. 7 pueden apreciarse las medidas registradas del perfil de oscilación y del perfil de oscilación amplificado. Como el láser de helioneón en $632.8 \mathrm{~nm}$ puede asociarse a un sistema de cuatro niveles ideal, la absorción desde el nivel de energía inferior de la transición láser puede considerarse despreciable. Entonces, analizando espectralmente la potencia cuando el tubo de helio-neón está apagado, medimos el perfil de oscilación (Fig. 7(a)). Y cuando el tubo de helio-neón está encendido (por tanto, bombeado), medimos el perfil de oscilación amplificado (Fig. 7(b)). Resulta necesario hacer notar que los picos que aparecen en la Fig. 7 tienen menor altura que la envolvente. Para explicarlo, hay que tener en cuenta que medimos la convolución de la transmisión espectral del Fabry-Pérot con el espectro de potencia del modo longitudinal. Como el modo longitudinal es mucho más estrecho, lo que medimos es la transmisión espectral del interferómetro. Si los modos longitudinales mantuviesen fijada su posición dentro del perfil de oscilación, el valor máximo de los picos coincidiría con el perfil de oscilación. Sin embargo, como los modos van corriendo a lo largo del perfil de oscilación, su posición se desplaza y sólo coincide un instante con el máximo de transmisión del Fabry-Pérot. Por tanto, justo cuando se captura la traza del osciloscopio, lo más probable es que la altura del pico esté por debajo del perfil de oscilación. Esta situación no afecta a la medida del perfil de oscilación porque se lleva a cabo durante periodos de tiempo muy largos.

Ajustando simultáneamente las dos envolventes que aparecen en la Fig. 7 a las Ecs. (8) y (13) se determinó que $X_{p}=1.35$ y que $\Delta v=0.88 \mathrm{GHz}$. Con estos valores, y según las Ecs. (10) y (12), el perfil de oscilación es más estrecho que el perfil de ganancia, ya que $\Delta v_{o s c}=0.39 \mathrm{GHz}$ y $\Delta v_{o s c, \max }=0.48 \mathrm{GHz}$. Como puede apreciarse en la Fig. 8, la calidad del ajuste es más que aceptable para la zona central del espectro. Sin embargo, en las zonas de menor potencia (bombeo muy cercano al umbral), los espectros medidos se alejan de los valores teóricos. Principalmente, esto es debido a que el modelo teórico que describe la potencia de salida de un láser en función del bombeo considera despreciable la emisión espontánea

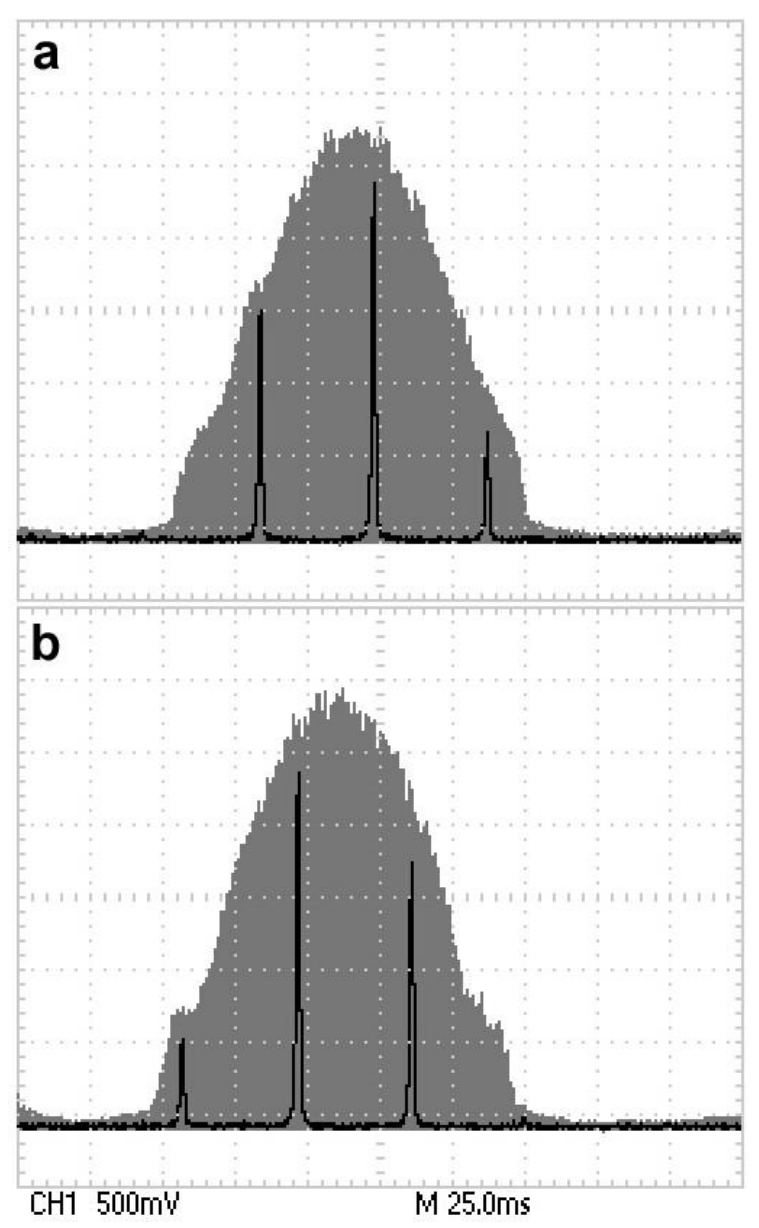

Fig. 7. Pantalla del osciloscopio digital con el tubo de helioneón sin bombear (a) y bombeado (b).

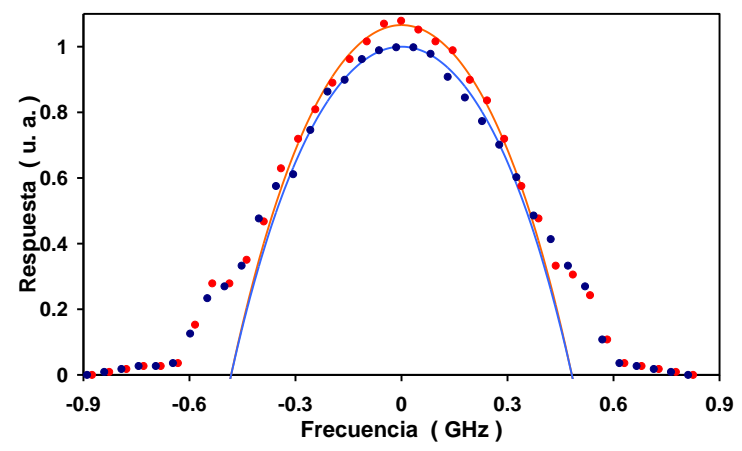

Fig. 8. Ajuste con el modelo teórico de los perfiles de oscilación (línea azul) y de oscilación amplificada (línea roja).

amplificada frente a la potencia láser emitida. Esta aproximación induce al modelo teórico a describir un comportamiento lineal con el bombeo y a asignar una potencia láser nula en el bombeo umbral. En general, esta aproximación está perfectamente justificada. Sin embargo, no es así cuando el bombeo es muy cercano al umbral, en cuyo caso la emisión espontánea 
amplificada supera claramente a la potencia láser emitida. Por tanto, la emisión espontánea amplificada es la causa de que la predicción teórica para el perfil de oscilación se aleje claramente de los valores experimentales encontrados.

El valor obtenido para la semianchura del perfil de ganancia está en concordancia con el aceptado en la bibliografía [11,12], $0.9 \mathrm{GHz}$. No obstante, para decidir finalmente el mejor par de valores de ajuste se tuvo que añadir la condición de que la ganancia media fuese 1.062, tal como se determinó en la Ref. [1]. El valor obtenido para $X_{p}$ es más difícil de comparar con la bibliografía. No obstante, es compatible con la Ref. [11], en la que se empleó un láser similar. Según los datos de esta referencia, $X_{p}$ debe ser inferior a 2.15 .

Finalmente, es necesario comentar que en este trabajo no se han incluido dos efectos que modificarían el perfil espectral de ganancia considerado. El primero de estos efectos es el hole-burning espectral [2], el cual no afecta a las medidas realizadas ya que no trabajamos con una resolución lo suficientemente alta. El segundo es la saturación. En nuestro caso, la intensidad óptica del láser utilizado es mucho menor que la intensidad de saturación del medio activo, la cual puede estimarse en $350 \mathrm{~mW} / \mathrm{mm}^{2}$ [12].

\section{Conclusiones}

Empleando material básico en un laboratorio docente dedicado a temas sobre láser $\mathrm{e}$ interacción luz-materia, es posible medir el perfil de oscilación de un láser de helio-neón mediante técnicas experimentales sencillas. Este perfil puede explicarse mediante modelos teóricos sencillos, pero que se ajustan satisfactoriamente a los resultados experimentales, permitiendo determinar las anchuras de los perfiles de ganancia y de oscilación.

La relación entre ambos perfiles es un tema básico en cualquier asignatura o curso sobre láser que puede ilustrarse perfectamente utilizando los resultados expuestos en este trabajo.

\section{Agradecimientos}

$\mathrm{Al}$ autor le gustaría agradecer al profesor Dr. D. José Miguel Álvarez Abenia sus comentarios y discusiones sobre este trabajo. 